\title{
Small-Diameter Vessels Reconstruction Using Cell Tissue- Engineering Graft Based on the Polycaprolactone
}

\author{
N. M. Yudintceva ${ }^{a, *}$, Yu. A. Nashchekina ${ }^{a}$, M. A. Shevtsov ${ }^{a, b}$, V. B. Karpovich ${ }^{b}$, G. I. Popov ${ }^{b}$, \\ I. A. Samusenko ${ }^{c}$, and N. A. Mikhailova ${ }^{a}$ \\ ${ }^{a}$ Institute of Cytology, Russian Academy of Sciences, St. Petersburg, 194064 Russia \\ ${ }^{b}$ Pavlov First State Medical University of St. Petersburg, St. Petersburg, 197022 Russia \\ ${ }^{c}$ The Nikiforov Russian Center of Emergency and Radiation Medicine, the Ministry of Russian Federation for Civil Defense, \\ Emergencies and Elimination of Consequences of Natural Disasters, St. Petersburg, 197082 Russia \\ *e-mail: yudintceva@mail.ru \\ Received January 21, 2021; revised February 4, 2021; accepted February 5, 2021
}

\begin{abstract}
Polycaprolactone (PCL) is widely applied for the construction of small-diameter tissue-engineered vascular grafts (TEGs) due to its biomechanical properties, slow degradation, and good biocompatibility. In the present study the TEG based on a tubular scaffold seeded with smooth muscle aortic cells (SMCs) in a rat abdominal aorta replacement model was tested. Polyester tubular scaffolds were generated by thermally induced phase separation and seeded with rat SMCs. To track the implanted SMCs in vivo, cells were labeled with superparamagnetic iron oxide nanoparticles (SPIONs). Histological evaluation of the migration of autologous endothelial cells (ECs) and formation of the endothelial lining was performed 4, 8, and 12 weeks after graft interposition. TEG demonstrated a high patency rate without any complications at the end of the 12-week period. The migration of ECs into the lumen of the implanted TEG and formation of the cell monolayer were already present at 4 weeks, as confirmed by histological analysis. The architecture of both neointima and neoadventitia were similar to those of the native vessel. SPION-labeled SMCs were detected throughout the TEG, indicating the role of these cells in the endothelization of scaffolds. The SMCseeded scaffolds demonstrated improved patency and biointegrative properties when compared to the acellular grafts.
\end{abstract}

Keywords: smooth muscle and endothelial cells, polycaprolactone, tubular scaffold, small diameter vessel, superparamagnetic iron oxide nanoparticles, tissue engineering graft

DOI: $10.1134 / \mathrm{S} 1990519 X 21060110$

\section{INTRODUCTION}

Bypass surgery is the main approach for the management of cardiovascular diseases associated with the obliteration of blood vessels. However, a lack of autologous vessels (veins or arteries) due to repeated operations or/and concomitant vascular pathology (i.e., atherosclerosis) highlights the necessity for the development of alternative vascular grafts. The generation of clinically applicable small-diameter neovessels $(<6$ $\mathrm{mm}$ ) as alternatives to autologous venous or arterial vascular substitutes is currently the subject of intense translational research (Li et al., 2014; Xu et al., 2017). In spite of mounting research data, a firm conclusion regarding the suitability of certain graft materials has not been yet established. In order to provide structural integrity and long-term patency, the ideal vascular graft should have an antithrombotic surface, resis-

Abbreviations: ECs-endothelial cells, PCL-Polycaprolactone, SEM-scanning electron microscopy, SMCs-smooth muscle cells, TEG-tissue engineering graft, TEM-transmission electron microscopy tance to aneurysm formation, and a low risk of complications such as intimal hyperplasia or calcifications.

Over the past decades, tissue-engineered grafts (TEG) employing different synthetic materials have been developed. Currently, biological scaffolds based on decellularized tissues (Hibino et al., 2015; Negishi et al., 2017), silk fibroin (Catto et al., 2015), hydrogels (Aussel et al., 2017), cell-synthesized extracellular matrix (ECM)-based blood vessel constructs (Rosellini et al., 2010) have been employed for vessel reconstruction with a variable success rate, which is partly defined by the chemical properties of the applied material (Pashneh-Tala et al., 2015). Most scaffolds currently employed in TEGs are generated from various biodegradable synthetic polymers (De Valence et al., 2012; Aussel et al., 2017). Polymers play a critical role in tissue engineering technology as they represent suitable scaffolds for application in cardiovascular surgery, mainly due to their practical advantages (e.g., ease of fabrication and implantation, storage, etc.). Furthermore, the rate of polymer degradation can be 
modulated by varying multiple factors including the polymer molecular weight and surface-area-to-volume ratio, among others. When compared to biological scaffolds, synthetic polymers demonstrate improved biomechanical properties that make them good candidates for graft interposition, particularly in vessels with high arterial blood pressure.

Poly- $\varepsilon$-caprolactone is a promising candidate for the development of scaffolds for TEGs due to its good biocompatibility, slow degradation rate, and excellent biomechanical properties (Fukunishi et al., 2016). Scaffold fabrication techniques also play an important role in the preparation of TEGs. The most frequently used techniques include electrospinning and particulate or porogen leaching (Hoch et al., 2014). However, application of a variety of synthetic materials can lead to various complications including thrombosis, intimal hyperplasia, and transplant calcification (Mel et al., 2008; Mehta et al., 2011).

One of the strategies to generate TEG is based on seeding biodegradable synthetic materials with two main types of cells: endothelial or/and smooth muscle cells (SMCs). The endothelium produces pleiotropic effects and plays an important role in the regulation of blood vessel homeostasis and vascular patency (Dharmashankar and Widlansky, 2010; Wilson et al., 2014). Incomplete endothelialization of TEGs, especially in the case of regenerating small-diameter blood vessels, can lead to various graft-related complications (e.g., thrombosis, suture leakage, pseudoaneurysms, etc.) (Stowell et al., 2018; Zhao et al., 2018). Scaffold relief was previously reported to have a significant effect on the formation of the endothelial lining. Many attempts have been made to form ridges and grooves on the surfaces of scaffolds (Wang et al., 2019) or to change the fiber stiffness (Stekelenburg et al., 2008; Bingcheng et al., 2020) in order to modulate the formation of the endothelial monolayer. However, an effective and quick solution to the problem of TEC endothelization has not yet been achieved.

SMCs also play an important role in vascular function via the regulation of vessel tonus and lumen diameter. Indeed, as demonstrated by De Valence et al., the failure of SMCs to penetrate and colonize the graft, with subsequent production of collagen and elastin, results in the formation of an impaired vessel wall with low vasomotricity and elasticity parameters that ultimately leads to long-term graft-related complications (De Valence et al., 2012). Vascular grafts seeded with autologous ECs normally do not evoke host immune responses. However, obtaining and cultivating autologous endothelial cells (ECs) presents various limitations, including a long cultivation period for cell expansion and decreased cell viability, which is largely dependent on the patient's age and comorbidities. Moreover, isolating autologous ECs requires a biopsy, which might be distressing for patients. Allogenic ECs are more suitable for graft seeding, but they are associated with an increased risk of immune rejection (Radke, et al., 2018).
In the present study, the abdominal aorta of rats was reconstructed by employing a tubular PCL scaffold seeded with allogenic rat SMCs. We hypothesized that following cell-seeded graft interposition, the host endothelium would form a cellular monolayer in the scaffold inner lumen that would improve the biocompatibility of the graft. To track SMCs in vivo, the latter were labeled with superparamagnetic iron oxide nanoparticles (SPIONs). Over a 12-week follow-up period, rat blood neovessels were histologically assessed for the presence of complications and for the generation of tissues. We demonstrated that the inner lumen of the TEG was completely covered with endothelial cells, and that SPION-labeled SMCs were present throughout the wall of the neovessel.

\section{MATERIALS AND METHODS}

Isolation of Smooth Muscle Cells (SMCs)

Cells were isolated from the abdominal aorta of Wistar rats $(n=6)$ using an enzymatic treatment method (Song et al., 2009). SMCs were cultivated with DMEM supplemented with $10 \%$ FBS and $50 \mu \mathrm{g} / \mathrm{mL}$ gentamicin. Cell cultures were kept in a humidified incubator $\left(5 \% \mathrm{CO}_{2}, 37^{\circ} \mathrm{C}\right)$ and culture medium was replaced every third day. Before the experiments, cells were harvested in the log phase of growth, and their viability was determined by $0.4 \%$ trypan blue exclusion. Cells from the four or six passages were used for experiments.

\section{Internalization of Nanoparticles by SMCS}

Superparamagnetic iron oxide $\left(\mathrm{Fe}_{3} \mathrm{O}_{4}\right)$ nanoparticles less than $50 \mathrm{~nm}$ in size were coated with dextran to increase their biocompatibility and reduce aggregation. The cells that reached a monolayer were incubated with nanoparticles at a concentration of $150 \mu \mathrm{g} / \mathrm{mL}$ for $24 \mathrm{~h}$ in a $\mathrm{CO}_{2}$ incubator. The medium was exchanged for a fresh one, and the cells on the slides were washed three times with PBS. Cell viability was assessed by staining with $0.4 \%$ Trypan blue solution (Biolot, Russia). Additionally, the cytotoxicity of nanoparticles was analyzed using the MTT method. The Vybrant ${ }^{\circledR}$ MTT kit was used in accordance with the manufacturer's protocol (Life Technologies, United States).

\section{Preparation and Modification of the Tubular PCL Scaffold}

As a first step, the matrix was prepared from melted sugar. The matrix presented a rod shape with a diameter of $1.1 \mathrm{~mm}$ and length of $60 \mathrm{~mm}$. The sugar rod was sequentially coated with three layers of a PCL polymer (Sigma-Aldrich, United States) in chloroform at a concentration of $2 \%$. Following air exposure for $24 \mathrm{~h}$ to fully evaporate the solvent, the tubular scaffold was rinsed in distilled water to remove the sugar crystals. The scaffold was sterilized using ozone gas at a concentration of $200 \mathrm{ppm}$ for $120 \mathrm{~min}$ at a relative humid- 
ity of $80 \%$. To enhance cell attachment, the tubular PCL scaffold was incubated in fibrinogen solution at a concentration of $0.1 \mathrm{mg} / \mathrm{mL}$ at $4^{\circ} \mathrm{C}$ overnight. For analysis of scaffold parameters and architecture, samples were examined using field-emission scanning electron microscopy (FE-SEM; JEOL, Japan). The mechanical tensile properties of samples were determined using a universal testing machine (Instron, United States).

\section{Seeding the PCL Scaffold with Nanoparticle-labelled SMCs (TEG)}

Approximately $20 \mu \mathrm{L}$ of cell suspension $(1 \times$ $10^{6}$ cells $/ \mathrm{mL}$ ) was slowly and gently injected into the lumen of the PCL scaffold using a micropipette. The scaffold was rotated every $30 \mathrm{~min}$ for a period of $4 \mathrm{~h}$ to facilitate uniform cell distribution. Then, DMEM/F12 culture medium supplemented with $10 \%$ FBS and gentamicin $(50 \mu \mathrm{g} / \mathrm{mL})$ was added until the scaffold was immersed, then it was incubated under $5 \% \mathrm{CO}_{2}$ at $37^{\circ} \mathrm{C}$. Cell distribution was evaluated by confocal microscopy (FV3000; Olympus, Germany) after 3 days and 3 weeks of the cultivation.

\section{Immunofluorescence Assay}

SMCs in chambered coverslips and seeded the PCL scaffolds with nanoparticle-labelled SMCs were washed three times with PBS and fixed with $10 \%$ neutral formalin (Sigma-Aldrich, United States) for 15 min. Samples were embedded in Tissue-Tek and stored at $-80^{\circ} \mathrm{C}$ for preparation of the cryosections. To permeabilize the cell membrane, $0.1 \%$ Triton X100 solution (Sigma-Aldrich, United States) was added for $15 \mathrm{~min}$. Cells were washed three times and incubated in $0.2 \%$ bovine serum albumin (BSA) for $1 \mathrm{~h}$ to block nonspecific binding. Sections were stained with $\alpha$ SMA (anti-alpha Smooth Muscle Actin antibody (Abcam, United States)) at a dilution $1: 250$ at $4{ }^{\circ} \mathrm{C}$ overnight. Rabbit immunoglobulins (Rabbit Antimouse IgG; Abcam, United States) conjugated with FITC were used as secondary antibodies at a dilution of $1: 500$. Incubation with the second antibodies was performed for $45 \mathrm{~min}$ at room temperature in the dark. After washing three times with PBS solution, nuclei were stained with DAPI (4',6-diamidino-2-phenylindole) dye (Thermo Fisher Scientific, United States) for $10 \mathrm{~min}$ and washed with PBS. Cells stained with only second antibodies were used as a negative control. Immunofluorescence assessment was done with a diode laser $(488 \mathrm{~nm})$, nuclei were detected with a diode laser $(405 \mathrm{~nm})$, and scanning with a reflected laser $(504 \mathrm{~nm})$ was used to analyze the intracellular localization of magnetic nanoparticles. We used a confocal microscope (Olympus FV3000) with an Olympus IX83 microscope confocal system (Olympus Corp., Japan).

\section{Surgical Technique}

Twelve male Wistar rats (weighing $350 \mathrm{~g}$ ) were obtained from the Rappolovo Laboratory Animal Nursery of the Russian Academy of Medical Sciences (St. Petersburg, Russia). The animals were divided into two groups $(n=6)$ : (1) an experimental group using a tubular PCL scaffold with nanoparticlelabeled SMCs (TEG); (2) a control group-acellular TEG. Inhalation anesthesia was employed, consisting of 3\% isoflurane (Laboratorios Karizoo, Spain) during the operation. Y-shaped laparotomy and mobilization of an infrarenal part of the abdominal aorta were performed (Fig. 1a). The 6-mm tubular PCL scaffold with nanoparticle-labeled SMCs (TEG) was used (Fig. 1b). The abdominal aorta was replaced with acellular TEG or the graft seeded with SMCs which were fixed to the edges of the defect with separate $9 / 0$ interrupted neilon sutures (Figs. 1c, 1d), respectively. TEG patency was assessed by the classical technique immediately and $30 \mathrm{~min}$ after implantation (Acland, 1972). Anticoagulants were not applied in the postoperative period. Animals received free access to water and a standard diet. Additionally, all animals received postoperative antibiotic prophylaxis (enrofloxacin, $5 \mathrm{mg} / \mathrm{kg}$ ) for 7 days.

\section{Histological Analysis}

At the designated time points (4, 8, and 12 weeks), animals were euthanized by $\mathrm{CO}_{2}$ asphyxiation and reconstructed vessels were extracted and fixed in $10 \%$ formalin. Further, the material undergoes standard processing in isopropyl alcohol and paraffin for the preparation of histological and histochemical preparations with a thickness of serial paraffin sections of 3$5 \mu \mathrm{m}$ which were stained with hematoxylin/eosin (H\&E). All samples were analyzed for infiltration of lymphocytes, histiocytes, epithelioid cells, and giant multinucleated cells. In addition, sections were stained with $\alpha$-SMA. Following incubation at room temperature for $30 \mathrm{~min}$ followed by several PBS washes, nuclei staining was carried out using Mayer's hematoxylin. The Mouse/Rabbit UnoVue ${ }^{\mathrm{TM}}$ HRP/DAB Detection System was used according to the manufacturer's protocol. A confocal laser scanning microscope (DM LS; Leica, Germany) was used for analysis. Microphotography was performed using a digital camera (Leica DC320, Germany). Vessel tissue of the intact rats was used as a control.

\section{Preparation of the Cryosections}

To detect cells labeled with nanoparticles, samples of reconstructed vessels were placed in Tissue-Tek (Sakura, Japan) and analyzed using confocal microscopy. Additionally cryosections were stained with $\alpha$ SMA (Abcam, United States) and rabbit anti-mouse FITC-labeled antibodies at a dilution of $1: 100$ and $1: 500$, respectively. Sections were additionally stained with DAPI and mounted in fluorescence 

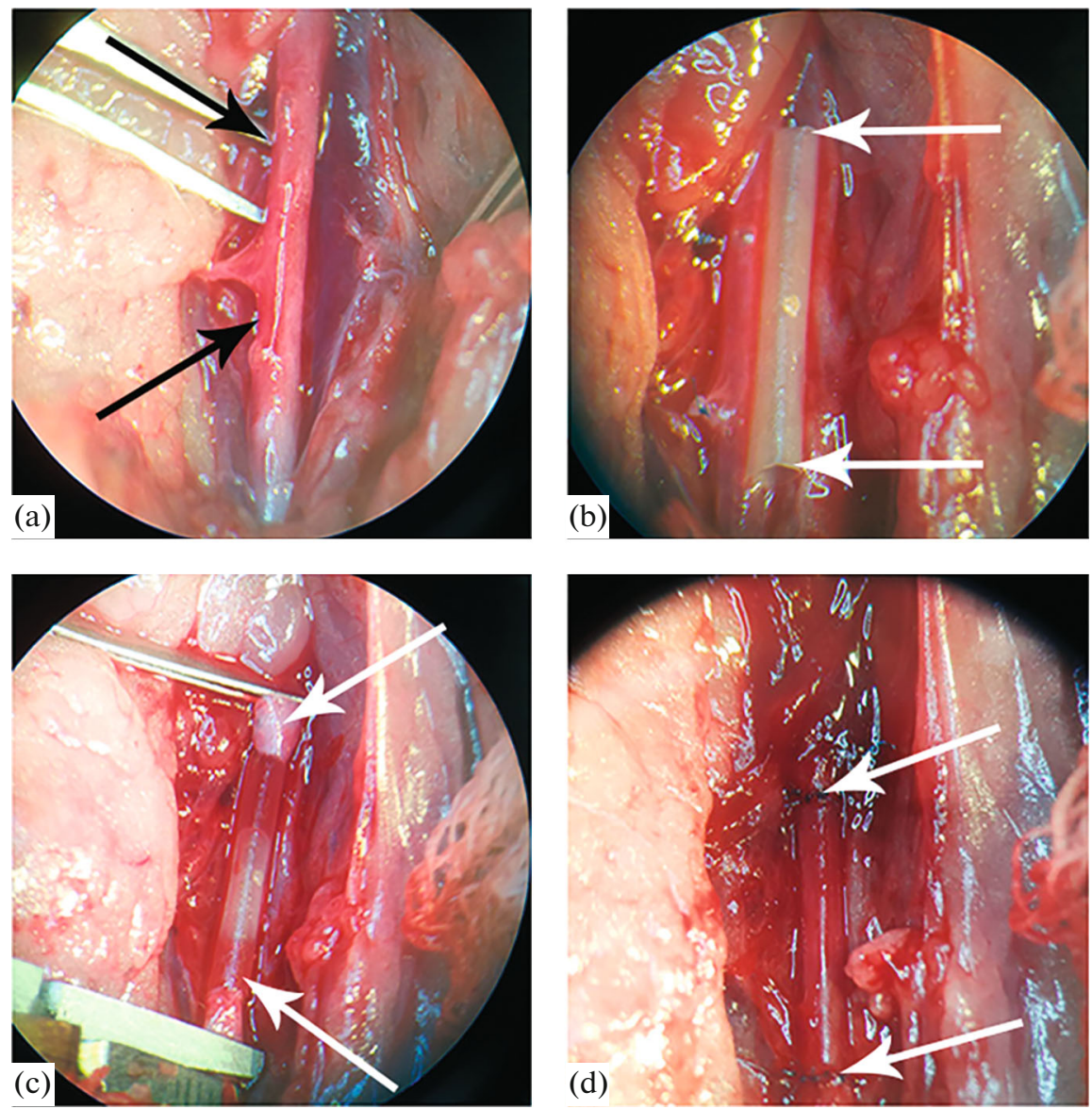

Fig. 1. Graft interposition in a rat abdominal replacement model. (a) Mobilization of an infrarenal part of the rat abdominal aorta (an aorta is indicated by black arrows), (b) creation of the TEG with a length of $6 \mathrm{~mm}$ (white arrows), (c) replacement of the abdominal aorta with TEG, and (d) aorta filled with blood.

mounting medium (Thermo Fisher Scientific, United States). Fluorescence images were captured with a confocal system (Olympus FV3000, Japan) using appropriate lasers (see Immunofluorescence Assay).

\section{Statistical Analysis}

The histological data and data of the evaluation of the mechanical properties of tubular scaffolds were subjected to statistical processing by methods of variation statistics using the Microsoft Excel program with determination of the mean (M) and error of the mean $(\mathrm{m})$, as were the comparison groups with the calculation of Student's $t$-test and level of significance $\alpha$ and confidence interval $p$ differences were considered significant at $p<0.05$.

\section{RESULTS AND DISSCUTION}

The SMCs isolated from the rat aorta were a homogeneous population with bipolar form (Fig. 2a) and were stained with $\alpha$-SMA antibodies (Fig. 2b). Superparamagnetic iron oxide nanoparticles had dimensions of less than $50 \mathrm{~nm}$ according to the TEM data (Fig. 2c). A high level of internalization of
SPIONs by cells was observed after 24 hours of incubation. SPIONs presented as red dots in cell cytoplasm surrounding but not penetrating the nucleus (Fig. 2d). Nanoparticles had no cytotoxic effect on SMCs after co-incubation for $24 \mathrm{~h}$ (data not shown). Our previous studies had confirmed the absence of the cytotoxic effect of nanoparticles used as a cell marker (Yudintceva et al., 2018) and for diagnostic purposes (Shevtsov et al., 2015).

The distribution of cells inside the tubular scaffold was evaluated using confocal microscopy. After 3 days of cultivation, the cells formed a monolayer on the inner surface of the scaffold, but they were already distributed over the entire thickness and over the entire length of the scaffold after 3 weeks (Fig. 2e,f, respectively).

According to the SEM data, the inner diameter of the PCL scaffold (Fig. 3a) was $1.1 \mathrm{~mm}$ (Fig. 3b) and wall thickness was $100 \mu \mathrm{m}$ (Fig. 3c). SMCs seeded inside of the tubular scaffold had a normal morphology (Fig. 3d).

The Young's modulus, tensile strength, and tensile deformation were determined for acellular tubular samples based on PCL, as well as for native rat aorta 
(a)

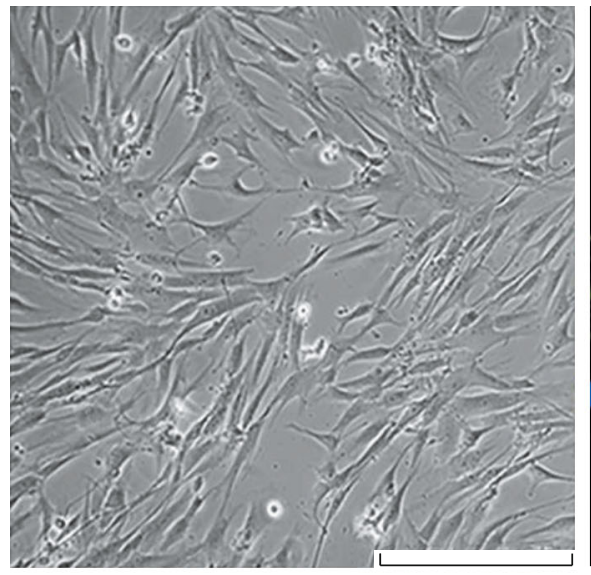

(c)

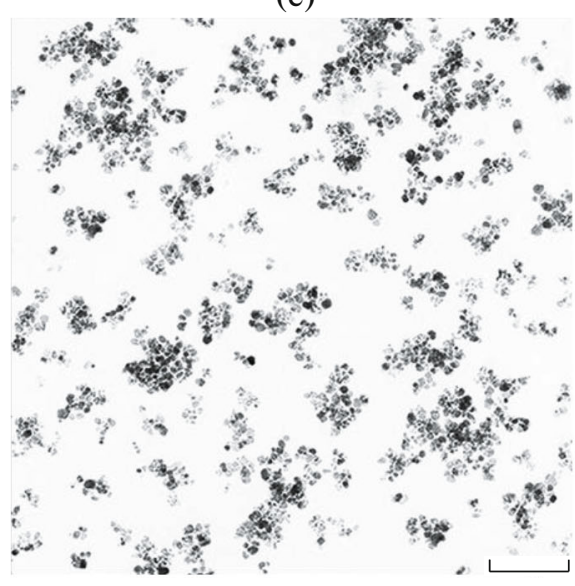

(e)

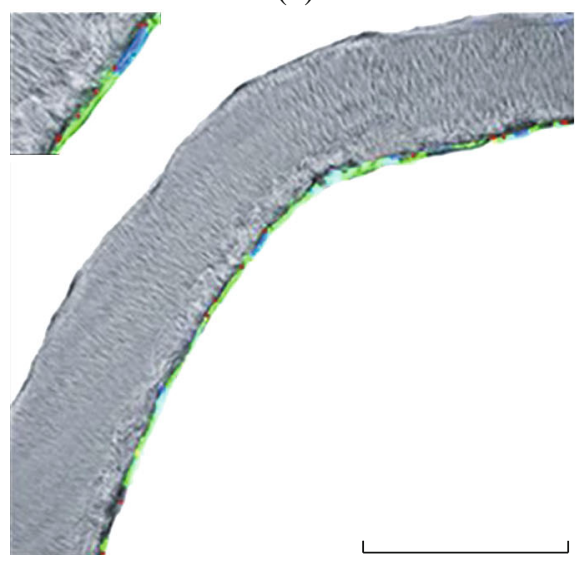

(b)

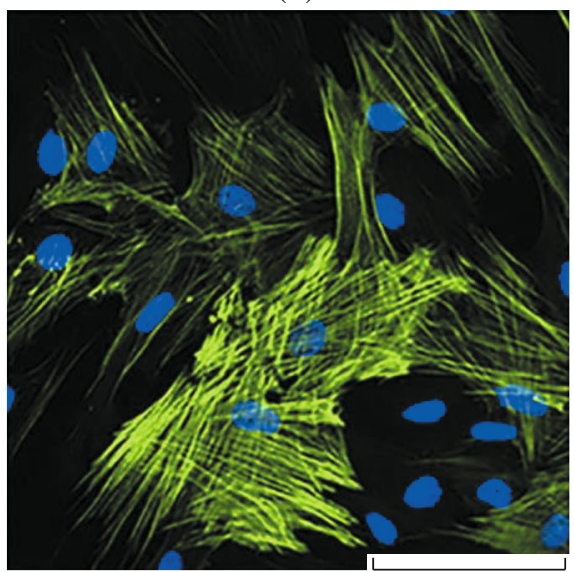

(d)

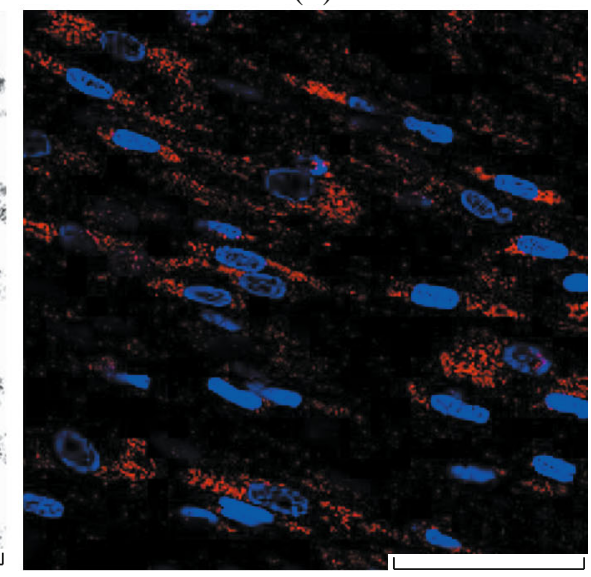

(f)

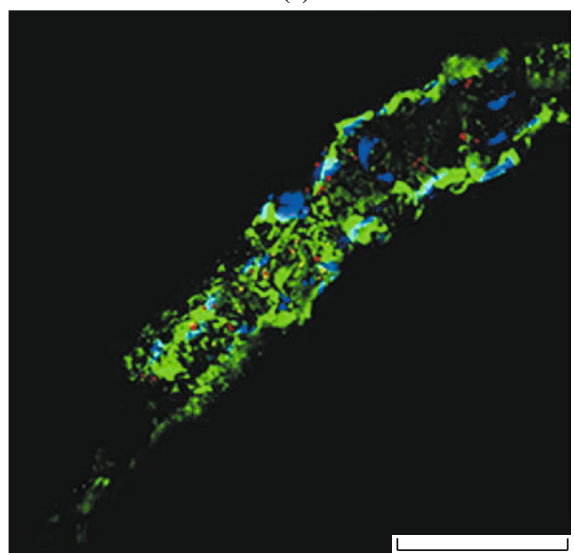

Fig. 2. SMCs derived from a rat abdominal aorta. (a) Morphology of the SMCs (Inverted Light Microscope Nikon Eclipse TS100 (Japan)) and (b) respective immunofluorescence assay of the specific $\alpha$ SMA protein expression (green). Scale bars are $200 \mu \mathrm{m}$. (c) TEM image of the SPIONs. Scale bar is $100 \mathrm{~nm}$. (d) Confocal microscopy images of SMCs coincubated with SPIONs. Nanoparticle-labeled SMCs seeded on the inner lumen of the scaffold after (e) 3 days and (f) 3 weeks of cultivation in vitro. SMCs were stained with specific antibodies ( $\alpha \mathrm{SMA})$, followed by secondary FITC-labeled antibodies (green). SPIONs were detected by reflected laser scanning at $504 \mathrm{~nm}$ (red), nuclei were stained with DAPI (blue). Scale bars are $100 \mu \mathrm{m}$.

samples. Despite the smaller wall thickness of the PCL scaffolds, the obtained data showed higher values for mechanical properties as compared to the rat aorta sample (Table 1).
In the control group, early graft thrombosis $(\leq 24 \mathrm{~h})$ was detected in five out of six animals using histological analysis. One in six animals survived till the end of the follow-up period of 12 weeks; however, subsequent 

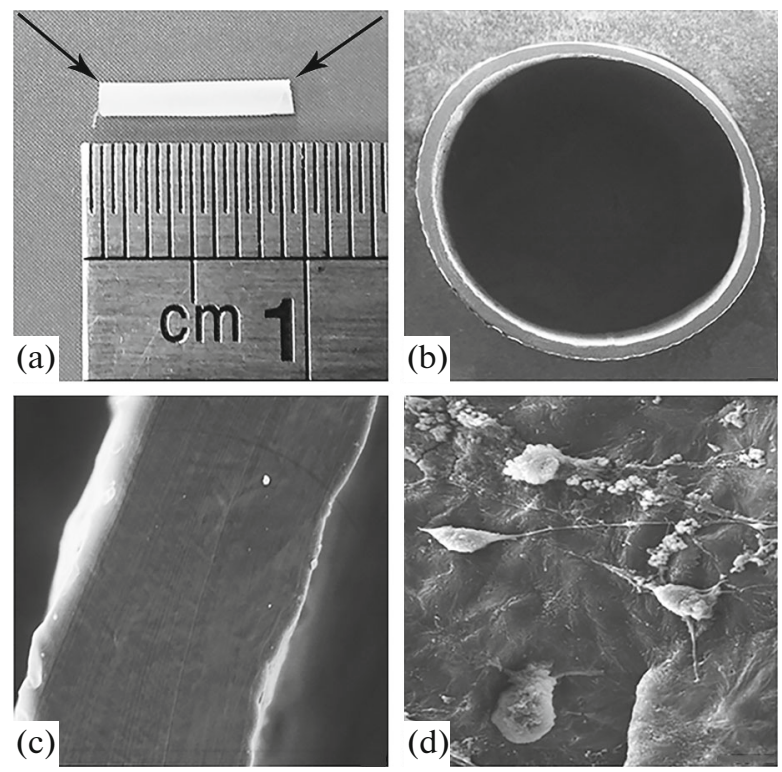

Fig. 3. Tubular PCL scaffold. (a) Macrophoto of the tubular PCL scaffold. (b, c) SEM of the cross, and longitudinal sections of the acellular tubular PCL scaffold. Scale bars are $100 \mu \mathrm{m}$. (d) SEM of the PCL scaffold following coincubation with SMCs for 24 hours. Scale bar is $10 \mu \mathrm{m}$.

evaluation confirmed late graft thrombosis, with recanalized thrombus soldered to the aortic wall in the aortic lumen (Fig. 4). In the experimental group that received cellular TECs no complications were detected. Experiments involving implantation of the grafts into rat abdominal aorta demonstrated that the grafts remained passable in all observed periods, the aorta was adherent to the graft, and no pathological influence (i.e., inflammation, hemorrhage) on the surrounding tissues was revealed. Neointima of the aorta presented as fibrous connective tissue with the inner elastic membrane covered with endothelium. There were no signs of hyperplasia of the neointima (Fig. 4). The vessel of the intact rat $(n=1)$ was used as a reference normal aorta control (Fig. 4).

Immunohistochemical (IHC) analysis clearly showed a tendency to form an ordered arrangement of smooth muscle fibers in the wall of the reconstructed vessels from 4-week period to 12-week period after surgery (Fig. 4). The material of the scaffold was not degraded and was visually verified in adventitia with weak inflammatory infiltration by single histiocytes, epithelioid cells, and giant polynuclear cells of foreign bodies. Subsequent confocal microscopy analysis demonstrated the presence of SPION-labeled cells throughout the graft over the analyzed 12-week period (Fig. 4). Furthermore, nanoparticle-labelled cells were also positively stained for $\alpha$-SMA.

Polyester PCL is a commonly used synthetic material for the reconstruction of small-caliber vascular grafts due to its excellent biocompatibility and suitable mechanical strength (Gao, 2016; Bouchet et al., 2019). The maximum elastic modulus is approximately $130 \mathrm{MPa}$ for human veins and $17 \mathrm{MPa}$ for arteries (Donovan et al., 1990; Porter et al., 1993). The tensile strength of human veins is about $6.5 \mathrm{MPa}$; in arteries, this value is lower (Stegemann et al., 2007). The strength and deformation stability of the generated tubular PCL scaffold were significantly higher than those of native blood vessels, indicating the possibility of their application as bioresorbable vascular grafts for tissue engineering applications (Table 1). However, in vitro engineering of small-diameter vascular grafts often results in various complications (e.g., stenosis, intimal hyperplasia, transplant calcification, etc.) when grafts are implanted in vivo. The long-term patency of small-diameter TEGs remains a great challenge in the field of cardiovascular tissue-engineering research due to the potential for luminal thrombosis. One approach for reducing the thrombogenicity of synthetic materials, although with limited success, is based on coating the luminal surface of the scaffold with anticoagulants (Devine et al., 2001; Ren et al., 2015). An alternative strategy to increase the biocompatibility of the graft and reduce the risk of complications is based on seeding the graft with vascular cells (Cui et al., 2019; Generali et al., 2019). An ideal cell source for seeding TEGs should be easily accessible and possess the ability to induce endothelialization. Vascular SMCs represent a promising source due to their ease of harvest and ability to facilitate endothelization (Arias et al., 2018; Hassan et al., 2018).

The current study demonstrated the ability of allogenic SMCs application in a PCL scaffold to induce the migration of autologous ECs in vivo. Histological studies clearly confirmed the biointegration of the SMC-seeded graft, which showed good patency when compared to the acellular scaffolds (Fig. 4). Colonization of the inner lumen of the graft by host ECs is supported by the in vitro data, which showed active EC migration on the surface of the SMC-coated scaffolds (Fig. 4). Furthermore, histological analysis demonstrated that the graft lumen was completely covered with endothelium monolayer, present from 4 weeks after graft interposition. Presumably, earlier migration

Table 1. Mechanical characteristics of the poly( $\varepsilon$-caprolactone) (PCL) scaffold

\begin{tabular}{l|c|c|c|c}
\hline \multicolumn{1}{c|}{ Sample } & Wall thickness, $\mu \mathrm{m}$ & Tensile strength, MPa & Young's modulus, MPa & Elongation, \% \\
\hline PCL scaffold & 100 & $23 \pm 2.36^{*}$ & $226 \pm 8.1^{*}$ & $800 \pm 66^{*}$ \\
Native rat aorta & 150 & $2.27 \pm 0.56$ & $17.3 \pm 5.1$ & $139 \pm 32$ \\
\hline
\end{tabular}

Data are presented as mean \pm SE. * $p$-value less than 0.05 was considered statistically significant. 
HL (H\&E)
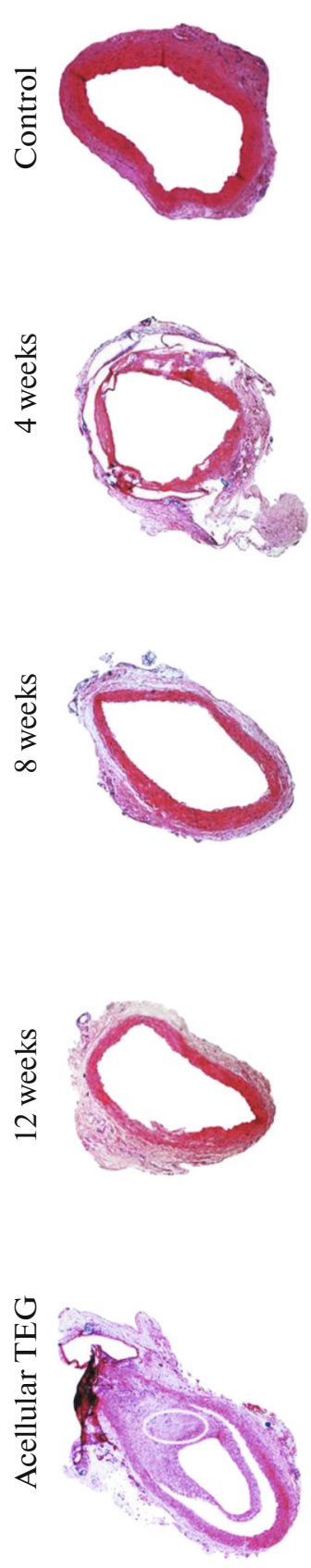

IHC ( $\alpha$ SMA $)$
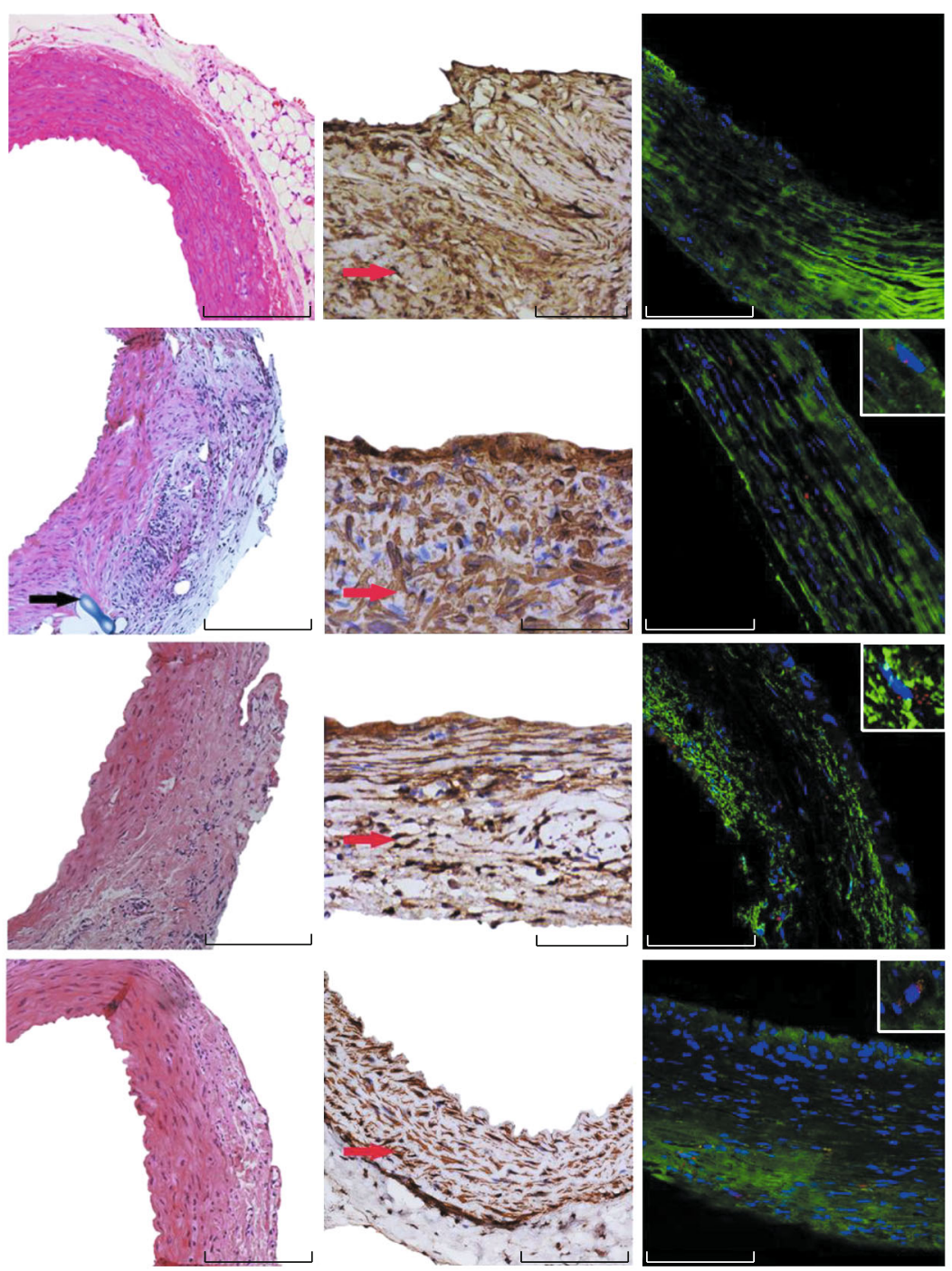

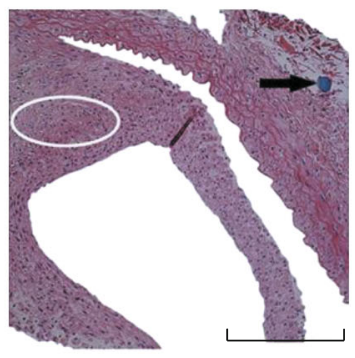

Fig. 4. Representative histological (HL), immunohistochemical (IHC), and immunofluorescence (IF) sections from the animals 4,8 , and 12 weeks after the graft interposition. The intact rat abdominal aorta was used as a reference control. Black arrows point to the material of the scaffold (H\&E stain). Red arrows point to the positive brown-stained SMCs (IHC). Assessment of the SPION-labeled SMCs in the reconstructed vessel wall was performed by immunofluorescence assay (right column). Labeled SMCs were additionally stained with specific anti- $\alpha$-SMA antibodies, followed by secondary FITC-labeled antibodies (green). SPIONs were detected by reflected laser scanning (red). Nuclei were stained with DAPI (blue). The area of vessel thrombosis is indicated by a white solid ellipse. Scale bars represent $100 \mu \mathrm{m}$.

of the autologous ECs prevented the formation of thrombosis, which occurred in the control group in the early postoperative period (Fig. 4). Previously, several studies reported the anticoagulant properties of ECs that contribute to the regulation of hemostasis (Van Hinsbergh et al., 2012; Yau et al., 2015). 
The obtained biomechanical and patency properties of the SMC-seeded PCL graft can compared to earlier studies that employed SMCs for seeding of decullarized (Neff et al., 2011) or synthetic (Cheng et al., 2008) scaffolds. The resorption rate of the PCL scaffold did not exceed the rate of ingrowth of cells and tissues inside it during the follow-up period, suggesting the possible use of this scaffold to generate neovessels with good long-term patency. Intriguingly, SPIONlabeled SMCs were detected throughout the graft over the analyzed period of 12 weeks (Fig. 4), which may indicate a role of these cells in the migration of ECs over the inner lumen of the graft.

Our data highlight the possible use of SMCs for engineering small-diameter vascular grafts, and demonstrated the successful generation of TEGs based only on one vascular cell type (SMCs) combined with a biodegradable polyester polymer (PCL).

\section{FUNDING}

Preparation of superparamagnetic nanoparticles of iron oxide and in vitro assessment of their cytotoxic properties was supported by the Russian Foundation for Basic Research no. 19-08-00024. In vivo experiments were supported by the Ministry of Science and Higher Education within the State assignment no. 0103-2019-0012. The present work was performed using equipment of the shared research facility "Vertebrate cell culture collection" supported by the Ministry of Science and Higher Education of the Russian Federation (Agreement no. 075-15-2021-683).

\section{COMPLIANCE WITH ETHICAL STANDARDS}

Conflict of interest. The authors declare that they have no conflict of interest.

Statement on the welfare of animals. The studies were conducted in accordance with GOST 33216-2014 "Rules for working with laboratory rodents and rabbits" and Protocol no. 1-208 of March 21, 2018 of the Ethical Committee of the Pavlov First State Medical University (St. Petersburg, Russia). All animals were treated according to the guidelines Federation of the European Laboratory Scientific Association.

\section{OPEN ACCESS}

This article is licensed under a Creative Commons Attribution 4.0 International License, which permits use, sharing, adaptation, distribution and reproduction in any medium or format, as long as you give appropriate credit to the original author(s) and the source, provide a link to the Creative Commons licence, and indicate if changes were made. The images or other third party material in this article are included in the article's Creative Commons licence, unless indicated otherwise in a credit line to the material. If material is not included in the article's Creative Commons licence and your intended use is not permitted by statutory regulation or exceeds the permitted use, you will need to obtain permission directly from the copyright holder. To view a copy of this licence, visit ttp://creativecommons. org/licenses/by/4.0/.

\section{REFERENCES}

Acland, R., Signs of patency in small vessel anastomosis, Surgery, 1972, vol. 72, p. 744.

Arias, S.L., Shetty, A., Devorkin, J., and Allain, J.P., Magnetic targeting of smooth muscle cells in vitro using a magnetic bacterial cellulose to improve cell retention in tissueengineering vascular grafts, Acta Biomater., 2018, vol. 77, p. 172.

Aussel, N.B., Thebaud, X., Berard, V., Brizzi, V., Delmond, S., Bareille, R., Siadous, R., James, C., Ripoche, J., Durand, M., Montembault, A., Burdin, B., Letourneur, D., Heureux, N.L., David, L., and Bordenave, L., Chitosan-based hydrogels for developing a small-diameter vascular graft: in vitro and in vivo evaluation, Biomed. $\mathrm{Ma}$ ter., 2010, vol. 12.

https://doi.org/10.1088/1748-605X/aa78d0

Bingcheng, Y., Yanbing, S., Tang, H., Wang, X., and Zhang, Y., Stiffness of the aligned fibers affects structural and functional integrity of the oriented endothelial cells, Acta Biomater., 2020.

https://doi.org/10.1016/j.actbio.2020.03.022

Bouchet, M., Gauthier, M., Maire, M., Ajji, A., and Lerouge, S., Towards compliant small-diameter vascular grafts: predictive analytical model and experiments, Mater. Sci. Eng. C. Mater. Biol. Appl., 2019, vol. 100, p. 715.

Catto, V., Fare, S., Cattaneo, I., Figliuzzi, M., Alessandrino, A., Freddi, G., Remuzzi, A., Tanzi, and M.C., Small diameter electrospun silk fibroin vascular grafts: mechanical properties, in vitro biodegradability, and in vivo biocompatibility, Mater. Sci. Eng. C. Mater. Biol. Appl., 2015, vol. 54, p. 101.

Cheng, S.T., Chen, Z.F., and Chen, G.Q., The expression of cross-linked elastin by rabbit blood vessel smooth muscle cells cultured in polyhydroxyalkanoate scaffolds, Biomaterials, 2008, vol. 29, no. 31, pp. 4187-4194.

Cui, H., Zhu, W., Huang, Y., Liu, C., Yu, Z., Nowick, M., Miao, S., Cheng, Y., Zhou, X., Lee, S., Zhou, Y., Wang, S., Mohiuddin, M., Horvath, K., and Zhang, L.G., In vitro and in vivo evaluation of $3 \mathrm{D}$ bioprinted small-diameter vasculature with smooth muscle and endothelium, Biofabrication, 2019, vol. 12.

https://dx.doi.org/10.1088\%2F1758-5090\%2Fab402c

De Valence, S., Tille, J.-Ch., Mugnai, D., Mrowczynski, W., Gurny, R., Moller, M., and Walpoth, B.H., Long term performance of polycaprolactone vascular grafts in a rat abdominal aorta replacement model, Biomaterials, 2012, vol. 33 , p. 38 .

Devine, C., Hons, B., and McCollum, C., Heparin-bonded dacron or polytetrafluoroethylene for femoropopliteal bypass grafting: a multicenter trial, J. Vasc. Surg., 2001, vol. 33 , p. 533.

Dharmashankar, K. and Widlansky, M.E., Vascular endothelial function and hypertension: insights and directions, Curr. Hypertens. Rep., 2010, vol. 12, p. 448.

Donovan, D.L., Schmidt, S.P., Townshend, S.P., Njus, G.O., and Sharp, W.V., Material and structural characterization of human saphenous vein, J. Vasc. Surg., 1990, vol. 12, p. 531.

Fukunishi, T., Best, C.A., Sugiura, T., Shoji, T., Yi, T., Udelsman, B., and Hibino, N., Tissue-engineered small di- 
ameter arterial vascular grafts from cell-free nanofiber $\mathrm{pcl} /$ chitosan scaffolds in a sheep model, PLoS One, 2016, vol. 11. e0158555.

https://doi.org/10.1371/journal.pone.0158555

Gao, C., Polymeric Biomaterials for Tissue Regeneration. From Surface/Interface Design 3D Constructs, 2016. https://www.springer.com/gp/book/9789811022920.

Generali, M., Casanova, E.A., Kehl, D., Wanner, D., Hoerstrup, S.P., Cinelli, P., and Weber, B., Autologous endothelialized small-caliber vascular grafts engineered from blood-derived induced pluripotent stem cells, Acta Biomater., 2019, vol. 97, p. 333.

Hassan, G.S., Jacques, D., D’Orleans-Juste, P., Magder, S., and Bkaily, G., Physical contact between human vascular endothelial and smooth muscle cells modulates cytosolic and nuclear calcium homeostasis, Can. J. Physiol. Pharmacol., 2018, vol. 96, p. 655.

Hibino, N., McConnell, P., Shinoka, T., Malik, M., and Galantowicz, M., Preliminary experience in the use of an extracellular matrix (CorMatrix) as a tube graft: word of caution, Semin. Thorac. Cardiovasc. Surg., 2015, vol. 27, p. 288 .

Hoch, E., Tovar, G.E., and Borchers, K., Bioprinting of artificial blood vessels: current approaches towards a demanding goal, Eur. J. Cardiothorac. Surg., 2014, vol. 46, p. 767.

Li, S., Sengupta, D., and Chien, S., Vascular tissue engineering: from in vitro to in situ, Wiley Interdiscip. Rev. Syst. Biol. Med., 2014, vol. 6, p. 61.

Mehta, R.I., Mukherjee, A.K., Patterson, T.D., and Fishbein, M.C., Pathology of explanted polytetrafluoroethylene vascular grafts, Cardiovasc. Pathol., 2011, vol. 20, p. 213.

Mel, A., Jell, G., Stevens, M.M., and Seifalian, A.M., Biofunctionalization of biomaterials for accelerated in situ endothelialization: a review, Biomacromolecules, 2008, vol. 9, p. 2969.

Neff, L.P., Tillman, B.W., Yazdani, S.K., Machingal, M.A., Yoo, J.J., Soker, S., and Christ, G.J., Vascular smooth muscle enhances functionality of tissueengineered blood vessels in vivo, J. Vasc. Surg., 2011, vol. 53, p. 426.

Negishi, J., Hashimoto, Y., Yamashita, A., Zhang, Y., Kimura, T., Kishida, A., and Funamoto, S., Evaluation of small-diameter vascular grafts reconstructed from decellularized aorta sheets, J. Biomed. Mater. Res. A, 2017, vol. 105, p. 1293.

Pashneh-Tala, S., MacNeil, S., and Claeyssens, F., The tissue-engineered vascular graft-past, present, and future, Tissue Eng. Part B. Rev., 2015, vol. 22, p. 68.

Porter, T.R., Taylor, D.O., Fields, J., Cycan, A., Akosah, K., Mohanty, P.K., and Pandian, N.G., Direct in vivo evaluation of pulmonary arterial pathology in chronic congestive heart failure with catheter-based intravascular ultrasound imaging, Am. J. Cardiol., 1993, vol. 71, p. 754.

Radke, D., Jia, W., Sharma, D., Fena, K., Wang, G., Goldman, J., and Zhao, F., Tissue engineering at the blood-contacting surface: a review of challenges and strategies in vascular graft development, Adv. Healthcare Mater., 2018. https://doi.org/10.1002/adhm.201701461

Ren, X., Feng, Y., Guo, J., Wang, H., Li, Q., Yang, J., and Li, W., Correction: surface modification and endothelialization of biomaterials as potential scaffolds for vascular tis- sue engineering applications, Chem. Soc. Rev., 2015, vol. 44, p. 5745 .

Rosellini, E., Vozzi, G., Barbani, N., Giusti, P., and Cristallini, C., Three-dimensional microfabricated scaffolds with cardiac extracellular matrix-like architecture, Int. J. Artif. Organs, 2010, vol. 33, p. 885.

Shevtsov, M.A., Nikolaev, B.P., Ryzhov, V.A., Yakovleva, L.Y., Dobrodumov, A.V., Marchenko, Y.Y., and Guzhova, I.V., Brain tumor magnetic targeting and biodistribution of superparamagnetic iron oxide nanoparticles linked with 70-kDa heat shock protein study by nonlinear longitudinal response, J. Magn. Magn. Mater., 2015, vol. 388, p. 123.

Song, H.J., Xue, W.J., Li, Y., Tian, X.H., Song, Y., Ding, X.M., and Li, Z.L., Improved islet survival and function with rat endothelial cells in vitro co-culture, Transplantology, 2009, vol. 41, p. 4302.

Stegemann, J.P., Kaszuba, S.N., and Rowe, S.L., Review: advances in vascular tissue engineering using protein-based biomaterials, Tissue Eng., 2007, vol. 13, p. 2601.

Stekelenburg, M., Rutten, MC, Snoeckx, H.E.H., and Baaijens, F.P., Dynamic straining combined with fibrin gel cell seeding improves strength of tissue-engineered smalldiameter vascular grafts, Tissue Eng. Part A, 2008, vol. 15, p. 1081.

Stowell, C.E.T., and Wang, Y., Quickening: translational design of resorbable synthetic vascular grafts, Biomaterials, 2018, vol. 173, p. 71.

Van Hinsbergh, V.W., Endothelium-role in regulation of coagulation and inflammation, Semin. Immunopathol., 2012, vol. 34, p. 93.

Wang, W., Xu, X., Li, Z., Kratz, K., Ma, N., and Lendlein, A., Modulating human mesenchymal stem cells using poly(n-butyl acrylate) networks in vitro with elasticity matching human arteries, Clin. Hemorheol. Microcirc., 2019, vol. 71, p. 277.

Wilson, H.K., Canfield, S.G., Shusta, E.V., and Palecek, S.P., Concise review: tissue-specific microvascular endothelial cells derived from human pluripotent stem cells, Stem Cells, 2014, vol. 32, p. 3037.

Xu, S., Lu, F., Cheng, L., Li, C., Zhou, X., Wu, Y., and Qi, Z., Preparation and characterization of small-diameter decellularized scaffolds for vascular tissue engineering in an animal model, Biomed. Eng. Online, 2017, vol. 16. https://doi.org/10.1186/s12938-017-0344-9

Yau, J.W., Teoh, H., and Verma, S., Endothelial cell control of thrombosis, BMC Cardiovasc. Disord., 2015, vol. 15, p. 130.

Yudintceva, N.M., Bogolubova, I.O., Muraviov, A.N., Sheykhov, M.G., Vinogradova, T.I., Sokolovich, E.G., and Shevtsov, M.A., Application of the allogenic mesenchymal stem cells in the therapy of the bladder tuberculosis, J. Tiss. Eng. Regen. Med., 2018, vol. 12. e1580.

https://doi.org/10.1002/term.2583

Zhao, Q., Cui, H., Wang, J., Chen, H., Wang, Y., Zhanget, L., and Wang, M., Regulation effects of biomimetic hybrid scaffolds on vascular endothelium remodeling of biomimetic hybrid scaffolds on vascular endothelium remodeling, ACS Appl. Mater. Interfaces, 2018, vol. 10, p. 23583.

Translated by N. Yudintceva 